\title{
15. Weltkongress der Physiotherapie
}

\begin{abstract}
"Moving physical therapy forward“ - unter diesem Motto trafen sich Physiotherapeuten aus aller Welt im kanadischen Vancouver. Alle vier Jahre lädt der Weltverband WCPT (World Confederation for Physical Therapy) ein, sich an der Entwicklung des Berufsbildes zu beteiligen, Therapietrends und das Neuste aus Wissenschaft und Forschung mitzubekommen und über Aus- und Weiterbildung zu diskutieren. Eine Veranstaltung, die bei allen Teilnehmern bleibende Eindrücke hinterließ.
\end{abstract}

P hysiotherapeuten, wohin das Auge blickte. Über fünf Tage war die kanadische Stadt Vancouver fest in physiotherapeutischer Hand. Mehr als 3.500 Therapeuten aus 89 Ländern tummelten sich vom 2.-6. Juni 2007 im Vancouver Convention and Exhibition Centre (VCEC) und nahmen an Veranstaltungen des 15. WCPT-Kongresses teil. Das direkt am Meer gelegene Kongresszentrum bot ausreichend Platz für 1.641 verschiedene Vorträge, Workshops und Diskussionsveranstaltungen, für eine Industrieausstellung mit 185 Ausstellern und über 900 Postern. Für jeden war etwas dabei, und da blieb es nicht aus, dass die Qual der Wahl einigen Kongressteilnehmer anzumerken war. Hektisches Blättern durch das umfangreiche Programmheft und schnelle Wechsel zwischen einzelnen Räumen sorgten aber auch dafür, dass man den Eindruck nicht los wurde, dass sich hier viel bewegt. Diese Bewegung wollte auch eine kleine Gruppe deutscher Teilnehmer erleben. Insgesamt 27 waren gekommen, um internationale Physiotherapieluft zu schnuppern oder um selbst einen Vortrag zu halten.

\begin{abstract}
„Mich hat die Dimension des Kongresses und das extrem umfangreiche Programm überwältigt. Man hat unter anderem erfahren, wie Therapeuten in anderen Ländern mit den gleichen Problemen umgehen, die wir haben.“
\end{abstract}

Rebekka Leonhardt, Physiotherapeutin aus Stuttgart

Politischer Auftakt • Dass Physiotherapeuten etwas bewegen können und sich als Gruppe politisch betätigen sollen, wurde auf der Eröffnungsveranstaltung deutlich. Dr. Martha Piper, einst Physiotherapeutin und heute eine der führenden Forscherinnen Kanadas auf dem Gebiet der Entwicklungsverzögerungen bei Kindern, hielt eine engagierte Rede. Sie forderte die Teilnehmer mit ihrem Vortrag „Physiotherapy - coming of age“ auf, sich weltweit als eine Gruppe zu

verstehen, die sich auch in politische Themen einmischt - und zwar nicht nur in gesundheitspolitische. Man solle als Gruppe die Stimme erheben. Und um gemeinsam stark zu sein, brauche es ihrer Ansicht nach bei jedem Einzelnen fünf wichtige Eigenschaften:

$>$ Hoffnung und den Glauben an eine gestaltbare Zukunft,

> Mut, sich einzugestehen, dass nicht alles, was Physiotherapeuten tun, stets wirksam und kosteneffektiv sein kann,

> Bereitschaft, hart zu arbeiten, um die Courage für das Einstehen gemeinsamer Ziele aufzubringen,

> Selbstbewusstsein und

> die Fähigkeit, Netzwerke zu bilden und zu nutzen.

Sie fragte die Physiotherapeuten im Publikum, ob sie auf die Frage: „Wofür steht die Physiotherapie?“, vorbereitet seien, und wünscht sich für die Zukunft, dass die Physiotherapie erwachsen wird und sich einmischt.

Das forderte am folgenden Tag auch Judith Heumann, eine engagierte Streiterin für die Bürgerrechte behinderter Menschen, in ihrem Keynote-Referat „From Patient to Citizen: Implications of the UN International Convention on the Rights of Persons with Disabilities“. Sie war als erste behinderte Frau Staatssekretärin in einer US-Regierung und ist heute erste Beraterin der Weltbank für die Anliegen behinderter Menschen. Judith
Downtown Vancouver: Die weißen Segel des Kongresszentrums sind weithin sichtbar.

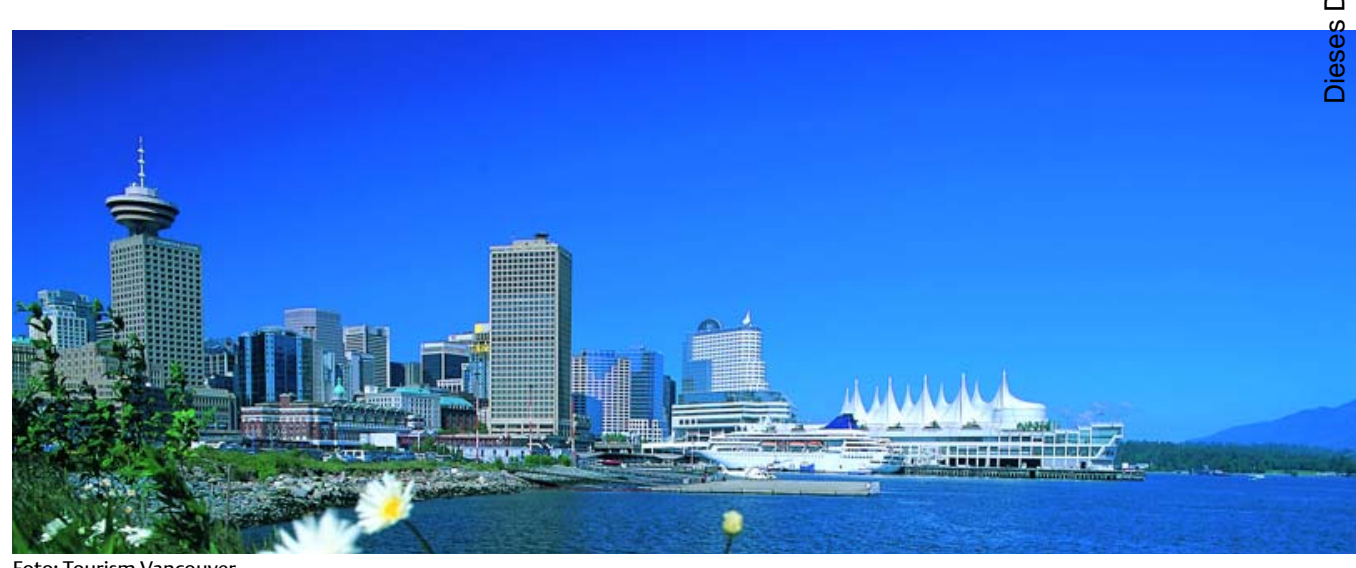

Heumann sieht gerade in der Gruppe der Physiotherapeuten eine gesellschaftspolitische Kraft, die an der Seite behinderter Menschen für deren Rechte kämpfen solle. Sie machte unmissverständlich deutlich, dass behinderte Menschen Physiotherapeuten nicht nur als Therapeuten sehen und sehen wollen, sondern sich auch deren Solidarität und Anwaltschaft wünschen. Wie Piper gab auch Heumann zu verstehen, dass sie sich mehr politisches Engagement wünscht.

„Der WCPT-Kongress hat mir viele
Möglichkeiten zum Austausch mit
Kollegen geboten und viele Denk-
anstöße gegeben. Es hat Spaß
gemacht, zum Reflektieren angeregt
zu werden und viel Motivation für
den Arbeitsalltag mitgegeben.“

Marisa Hoffmann, Physiotherapeutin aus Mainz

\begin{abstract}
Abgelehnte Visa verhinderten Teilnahme • Dass in umgekehrter Richtung Politik auch Einfluss auf Physiotherapeuten haben kann, mussten über 50 Therapeuten im Vorfeld des Kongresses erfahren. Iran und Afghanistan beispielsweise, die von neuen, strikteren Einreiseregeln betroffen sind, konnten keinen offiziellen Vertreter zum General Meeting der WCPT-Mitgliedsländer schicken. Und in Nigeria schien es besonders schwer zu sein: Über 40 nigerianischen Physiotherapeuten wurde ein Visum verweigert.

Dabei hatte man eine Menge getan, um möglichst vielen Therapeuten aus Entwicklungsländern die Kongressteilnahme zu ermöglichen. 2005 gründete sich eine Projektgruppe, die sich das Sammeln von Spenden zur Aufgabe gemacht hat. Neben Organisa-
\end{abstract}


weniger als einen Monat vorhanden waren. Zudem waren diese Patienten eher jünger, hatten einen höheren Bildungsabschluss und hatten bereits Erfahrung mit Physiotherapie gemacht. $10 \%$ der Patienten, die direkt zum Physiotherapeuten kamen, mussten nicht mehr weiterbehandelt werden. Es war also nur eine Behandlungseinheit nötig, um die Beschwerden der Patienten zu beheben. Zudem erhielten diese Patienten weniger Behandlungseinheiten als Patienten, die vom Arzt überwiesen wurden.

In den Jahren 2005 und 2006 gab es durch die Einführung des Direktzugangs entgegen den Befürchtungen der Politik keinen Anstieg der Patientenzahlen, die Physiotherapie erhielten. Allgemeinärzte konnten durch das neue System des Direktzugangs entlastet werden. Sie sehen weniger Patienten mit Beschwerden am Bewegungssystem, und nach den ersten Daten ist zu vermuten, dass man mit dem Direktzugang Kosten im Gesundheitssystem einsparen kann. Dies ist für die Niederlande allerdings noch nicht statistisch endgültig belegt.

Die guten Ergebnisse aus unserem Nachbarland lassen hoffen, dass auch in Deutsch- land die Einführung des Direktzugangs in naher Zukunft möglich wird.

\begin{abstract}
„Die WCPT-Kongresse zählen zu den Höhepunkten in meiner 25-jährigen beruflichen Laufbahn. Auch der Kongress in Vancouver war mal wieder eine Reise wert. Leider war die deutsche Delegation trotz erheblicher Fortschritte in der nationalen Akademisierung der Berufsbildung nur spärlich vertreten.“
\end{abstract}

$$
\begin{array}{r}
\text { Dietmar Seidenspinner, } \\
\text { Physiotherapeut aus Metzingen }
\end{array}
$$

Weltkongress 2011 in Amsterdam • Mit 27 Teilnehmern war Deutschland verglichen mit anderen Ländern leider schwach vertreten. Aus der Schweiz beispielsweise waren 67 und aus den Niederlanden 61 Teilnehmer angereist, von denen viele ihre Arbeiten in Vorträgen oder auf Postern vorstellten. Selbst Taiwan war mit 35 Therapeuten vertreten und Südafrika mit 38. Auf einem spontanen Treffen der deutschen Therapeuten, zu dem der ZVK geladen hatte, versuchte man
Gründe für diese Zahlen zu finden. Es gebe keine Kongresskultur, und man sei hierzulande einfach nicht gewohnt, in 20-minütigen Vorträgen Studienergebnisse präsentiert zu bekommen, vermuteten die meisten. Außerdem schrecke viele wahrscheinlich die englische Sprache ab, und Kanada sei leider nicht um die Ecke. Umso erfreulicher war für alle die Nachricht, dass der nächste Weltkongress 2011 in Amsterdam stattfinden wird. Ulrike Steinecke, Vorsitzende des ZVK, stellte in Aussicht, dass dort einige Vorträge auch in deutscher Sprache gehalten werden könnten.

Ob Englisch oder Deutsch, die anwesenden Therapeuten waren sich einig, dass sie alles dafür tun werden, in Amsterdam wieder dabei zu sein. Rebekka Leonhardt, Physiotherapeutin aus Stuttgart, machte deutlich, warum: „Für mich war der Kongress eine tolle Erfahrung mit der einmaligen Möglichkeit, sich mit Kollegen aus der ganzen Welt auszutauschen und den eigenen Blickwinkel zu erweitern - noch dazu in einer traumhaften Stadt. So etwas inspiriert und motiviert unheimlich für die tägliche Arbeit.“

Andrea Niesert, Fritz M. Koller, Rosi Haarer-Becker

\section{NACHGEHAKT}

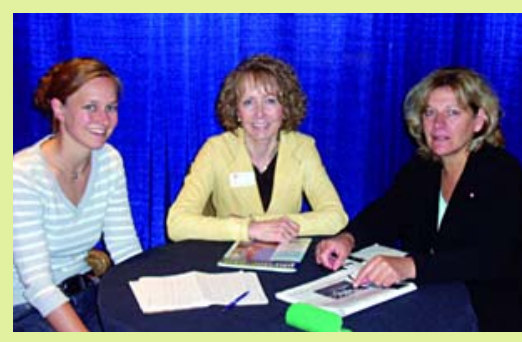

Pamela C. Fralick (Mitte) ist Geschäftsführerin der Canadian Physiotherapy Association (CPA) und war für die Organisation des WCPT-Kongresses 2007 verantwortlich. Im Interview berichtet sie, welchen Stellenwert die Veranstaltung für das Gastgeberland hat.

\section{> Seit wann sind Sie mit der Organisation} des WCPT-Kongresses 2007 beschäftigt? Direkt nach unserer Nominierung auf dem Weltkongress 2003 in Barcelona begannen wir mit der Planung des Programms und starteten verschiedene Aktivitäten. So haben wir beispielsweise einen virtuellen Fackellauf ins Leben gerufen: Die Fackel sollte von St. Johns im Osten bis nach Vancouver im Westen entlang des Trans Canada Trail getragen werden. Kanadische
Physiotherapeuten konnten zum Beispiel zu Fuß, per Fahrrad, Kanu oder Ski Kilometer sammeln und sie auf der virtuellen Karte einzeichnen lassen. Diese Idee wollen die Niederländer jetzt wahrscheinlich für den Weltkongress 2011 übernehmen und die Fackel in den nächsten vier Jahren von Therapeuten durch Europa tragen lassen.

Unser Ziel im Jahr 2007, 2.007 kanadische Physiotherapeuten nach Vancouver zu lotsen, haben wir trotz aller Aktivitäten dennoch leider nicht erreicht. Mit 1.100 Teilnehmern aus dem eigenen Land sind wir aber zufrieden.

\section{> Hat der Kongress an sich und haben} Aktionen wie der Fackellauf einen Einfluss auf die Präsenz der Physiotherapie in den Medien gehabt?

Ja, sehr. Die Physiotherapie war in den letzten Tagen in den Medien präsenter als je zuvor: Circa 30 Meldungen gab es im Radio, TV und in den Zeitungen. Der Präsident des Verbandes aus British Columbia beispielsweise hat ein 30-minütigen Fernsehinterview gegeben.
> Die Teilnehmer wurden aufgefordert, sich als Berufsgruppe politisch und nicht nur berufspolitisch zu engagieren. War Ihnen das ein besonderes Anliegen?

Ja, das haben wir ganz bewusst gesteuert: Wir wollen, dass die Physiotherapie in der Gesellschaft als gestaltende Gruppe wahrgenommen wird. Es ist wichtig, dass wir nun beginnen, über die Grenzen unseres Berufs hinauszugucken.

> Haben Sie eine Botschaft, die Sie gerne den deutschen Physiotherapeuten übermitteln möchten?

Haltet inne, hebt den Kopf und schaut, was in der Welt passiert! Auf einem Weltkongress können wir uns therapeutisch verbessern und eine starke Gruppierung in der Welt werden, die eine gemeinsame Stimme nach außen hat. Jeder Therapeut profitiert davon: Man trifft Kollegen aus aller Welt, und gerade in kleinen Ländern entstehen innovative Ideen, von denen man lernen kann.

Das Gespräch führten Rosi Haarer-Becker und Andrea Niesert. 